\title{
Senescent T cell population in Glioblastoma
}

\author{
Leo Song1 ${ }^{1}$, Mario Henriquez ${ }^{2}$, Sreenivasulu Chintala $\mathrm{PhD}^{2}$, Mahua Dey $\mathrm{MD}^{2}$ \\ ${ }^{1}$ Indiana University School of Medicine, Indiana, USA, ${ }^{2}$ Department of \\ Neurosurgery, Indiana University School of Medicine, Indiana, USA
}

Background and Hypothesis: Glioblastoma (GBM) is the most common primary brain tumor in adults and has a median overall survival of 20.6 months. Intracranial location, infiltrative growth with near $100 \%$ recurrence and the blood brain barrier are challenges which make treating GBM difficult. CD8 ${ }^{+} \mathrm{T}$ cells (CTLs) are largely responsible for mediating anti-tumor responses and thus, are an endpoint to most immunotherapies. However, the immunosuppressive tumor microenvironment (TME) hampers T cell effectiveness and causes exhaustion and senescence. Senescent CTLs $\left(C D 28^{-} \mathrm{CD} 57^{+}\right)$can result from DNA damage which causes loss of CD28. Lack of CD28 renders CTLs insensitive to immune signals and result in loss of cytotoxic functions. Thus, we hypothesize that CTLs have lost CD28 expression and are becoming senescent in the TME of GBM patients.

Experimental Design or Project Methods: CD8 and CD28 RNA expression data was pulled from TCGA in glioma and normal brain patients and a Pearson's correlation was conducted. Lymphocytes were isolated from patient tumor brain and blood and using flow cytometry, CD3+ CD8+ T cells were identified and stained for CD28, CD57, PD-1, and TIM-3.

Results: RNA sequencing data from TCGA database on CD8 and CD28 expression showed a negative correlation in GBM patients compared to normal patients. Majority of CD8+ lymphocytes from patient brain had lost CD28+ expression compared to blood.

Conclusion and Potential Impact: The increased senescent T cell population in GBM suggests that the TME has a mechanism for cytotoxic $T$ cell immunosuppression. Preventing CD8+ T cell senescence in GBM can improve current immunotherapies by promoting activation of tumor infiltrating lymphocytes. 